\title{
STRONGLY EXPOSED POINTS IN BASES FOR THE POSITIVE CONE OF ORDERED BANACH SPACES AND CHARACTERIZATIONS OF $l_{1}(\Gamma)$
}

\author{
by IOANNIS A. POLYRAKIS
}

(Received 27th May 1985)

\section{Introduction}

The study of extreme, strongly exposed points of closed, convex and bounded sets in Banach spaces has been developed especially by the interconnection of the RadonNikodým property with the geometry of closed, convex and bounded subsets of Banach spaces [5], [2]. In the theory of ordered Banach spaces as well as in the Choquet theory, [4], we are interested in the study of a special type of convex sets, not necessarily bounded, namely the bases for the positive cone. In [7] the geometry (extreme points, dentability) of closed and convex subsets $K$ of a Banach space $X$ with the Radon-Nikodým property is studied and special emphasis has been given to the case where $K$ is a base for a cone $P$ of $X$. In [6, Theorem 1], it is proved that an infinite-dimensional, separable, locally solid lattice Banach space is order-isomorphic to $l_{1}$ if, and only if, $X$ has the Krein-Milman property and its positive cone has a bounded base.

In this paper (Section 3) we study the existence of strongly exposed points in a base $B$ for a cone $P$ of a Banach space $X$ and we characterize the strongly exposing functionals. It is proved (Theorem 3.1) that the existence of strongly exposed points in a base $B$ for $P$ is closely connected with the existence of a bounded base for the cone $P$.

In Section 4 we prove a similar result to [6, Theorem 1] for the space $l_{1}(\Gamma)$, (Theorem 4.1). Afterwards we prove that if a Banach space $X$ ordered by the closed, generating cone $P$ has the R.D.P. then $X$ is order-isomorphic to $l_{1}(\Gamma)$ if, and only if, $P$ has the Krein-Milman property and $\operatorname{sep}(B) \neq 0$, for at least one base $B$ for $P$, (Proposition 4.2).

\section{Notations and definitions}

Let $X$ be a normed space, $K$ a convex subset of $X$ and $x_{0} \in K$. We say that $x_{0}$ is an exposed point of $K$ if there exists a continuous linear functional $g$ of $X\left(g \in X^{*}\right)$ such that $g\left(x_{0}\right)>g(x), \forall x \in K \backslash\left\{x_{0}\right\}$. In this case we say that $g$ exposes $x_{0}$ in $K$. We say that $x_{0}$ is a strongly exposed point of $K$ if there exists $g \in X^{*}$ which exposes $x_{0}$ in $K$ and for each sequence $\left(x_{v}\right)$ of $K, g\left(x_{v}\right) \rightarrow g\left(x_{0}\right)$ implies $x_{v} \rightarrow x_{0}$. In this case we say that $g$ strongly exposes $x_{0}$ in $K$. We denote by $\operatorname{ep}(K), \operatorname{sep}(K)$ the set of extreme, strongly exposed points of $K$. For each $A \subseteq X$ we denote by $\bar{A}$ the closure of $A$ and by $\bar{c} \bar{A}$ the closed convex hull of $A$. Let $X$ be ordered by the cone $P$. $x \in P \backslash\{0\}$ is an external point of $P$. 
$x \in \operatorname{EP}(P)$, if for each $y \in P, 0<y<x$ implies $y=\lambda x$. A subset $B$ of $P$ is a base for $P$ if there exists a strictly positive linear functional $f$ of $X$ such that $B=\{x \in P \mid f(x)=1\}$. Then we say that the base $B$ is defined by the functional $f$. The cone $P$ is generating if $X=P-P$. We say that $P$ is well-based if there exists a bounded base $B$ for $P$ and $0 \notin \bar{B}$. The space $X$ is a locally solid linear lattice if $X$ is a linear lattice and there exists a real number $a>0$ such that for each $x, y \in X,|x| \leqq|y|$, implies $\|x\| \leqq a\|y\|$. A linear functional $f$ of $X$ is uniformly monotonic if there exists a real number $a>0$ such that $f(x) \geqq a\|x\|, \forall x \in P$.

It is easy to show that $g$ strongly exposes $O$ in $P$ if, and only if, $-g$ is uniformly monotonic and therefore that $0 \in \operatorname{sep}(P)$ if, and only if, $P$ is well-based. $X$ is orderisomorphic to an ordered normed space $Y$ if there exists an isomorphism $T$ of $X$ onto $Y$ and $T, T^{-1}$ are positive. An ordered linear space $X$ has the Riesz decomposition property (R.D.P.) if for any three positive elements $x, y, z$ of $X$ with $x \leqq y+z$ there exist $x_{1}, x_{2} \in X$ such that $0 \leqq x_{1} \leqq y, 0 \leqq x_{2} \leqq z$ and $x=x_{1}+x_{2}$. Each linear lattice has the R.D.P.

\section{Strongly exposed points in a base for a cone}

Proposition 3.1. Let $X$ be a normed space and $K$ be an unbounded, convex subset of $X$. If $g$ strongly exposes the point $x_{0}$ in $K$, then $g\left(x_{v}\right) \rightarrow-\infty$, for each sequence $\left(x_{v}\right)$ of $K$ with $\left\|x_{v}\right\| \rightarrow+\infty$.

Proof. Let $\rho \in \mathbb{R}$ such that $\left\|x_{0}\right\|<\rho$ and $\left(x_{v}\right)$ be a sequence of $K$ with $\lim _{v \rightarrow \infty}\left\|x_{v}\right\|=$ $+\infty$. Then there exists a sequence $\left(y_{v}\right)$ of $K$, such that

$$
\left\|y_{v}\right\|=\rho \text { and } y_{v}=\lambda_{v} x_{0}+\left(1-\lambda_{v}\right) x_{v}, \quad \lambda_{v} \in(0,1) .
$$

Then

$$
\rho=\left\|y_{v}\right\| \geqq\left|\lambda_{v}\left\|x_{0}\right\|-\left(1-\lambda_{v}\right)\left\|x_{v}\right\|\right|
$$

and therefore $\lambda_{v} \rightarrow 1$.

If $\left(g\left(x_{k_{v}}\right)\right.$ is a bounded subsequence of $\left(g\left(x_{v}\right)\right)$, then

$$
g\left(y_{k_{v}}\right) \rightarrow g\left(x_{0}\right)
$$

and therefore $y_{k_{v}} \rightarrow x_{0}$. This is a contradiction. Hence $g\left(x_{v}\right) \rightarrow-\infty$ because $g\left(x_{v}\right) \leqq g\left(x_{0}\right)$.

Let $X$ be a normed space, $K$ a convex subset of $X, u \in X^{*}$ such that $u(K)=\{1\}$ and $\lambda \in \mathbb{R}$. By a simple computation we have:

$\left(S_{1}\right)$ A functional $g \in X^{*}$ strongly exposes $x_{0}$ in $K$ if, and only if, $g-\lambda u$ strongly exposes $x_{0}$ in $K$.

Since $\left(g-g\left(x_{0}\right) u\right)\left(x_{0}\right)=0$, we have that

$\left(S_{2}\right) x_{0}$ is a strongly exposed point of $K$ if, and only if, there exists $g \in X^{*}$ such that $g$ strongly exposes $x_{0}$ in $K$ and $g\left(x_{0}\right)=0$. 
If $g$ is as in $\left(S_{2}\right)$, then $g(x) \leqq 0$ for each $x \in K$ and we shall say that $g$ is a negativestrongly exposing functional of $x_{0}$ in $K$. Let $X$ be ordered by the cone $P$ and $x_{0}$ be an extremal point of $P$. We shall say that $x_{0}$ has a continuous projection $P_{x_{0}}$ (or that $x_{0}$ is an extremal point of $P$ with continuous projection $P_{x_{0}}$ ) if, and only if, $P_{x_{0}}$ is a linear, continuous, positive projection of $X$ onto $\left[x_{0}\right]$ such that $P_{x_{0}}(x) \leqq x$, for each $x \in P$.

Let $x_{0}$ be an extremal point of $P$ with continuous projection $P_{x_{0}}$. We denote by $\rho\left(x_{0}, \cdot\right)$ the continuous linear functional of $X$, defined by the formula

$$
P_{x_{0}}(x)=\rho\left(x_{0}, x\right) x_{0}, \quad x \in X
$$

Also for each $h \in X^{*}$ we denote by $h_{x_{0}}$ the functional

$$
h_{x_{0}}(x)=h\left(P_{x_{0}}(x)-x\right), \quad x \in X .
$$

If $B$ is a base for $P$ defined by $f \in X^{*}$ and $x_{0}^{\prime}=\lambda x_{0} \in B$, then we have:

$\left(P_{1}\right) P_{x_{0}}(x)<x_{0}^{\prime}$, for each $x \in B \backslash\left\{x_{0}^{\prime}\right\}$,

$\left(P_{2}\right)$ for each sequence $\left(x_{v}\right)$ of $B, f\left(P_{x_{0}}\left(x_{v}\right)\right) \rightarrow 1$ implies $P_{x_{0}}\left(x_{v}\right) \rightarrow x_{0}^{\prime}$,

$\left(P_{3}\right)$ for each strictly positive, continuous linear functional $h$ of $X, h_{x_{0}}$ exposes $x_{0}^{\prime}$ in $B$ and $h_{x_{0}}\left(x_{0}^{\prime}\right)=0$.

The statement $\left(P_{1}\right)$ is true because $x_{0}^{\prime} \leqq P_{x_{0}}(x) \leqq x$ implies $f\left(x-x_{0}^{\prime}\right)=0$ hence $x=x_{0}$.

Definition 3.1. A normed space $X$ ordered by the cone $P$ has the continuous projection property (C.P.P.), if $x \in \mathrm{EP}(P)$ implies that $x$ has a continuous projection.

Proposition 3.2. Let $X$ be a normed space ordered by the cone P. Then:

(i) $X$ has the C.P.P. if, and only if, $Y=P-P$ has the C.P.P.,

(ii) if $X$ is a locally solid linear lattice then $X$ has the C.P.P.,

(iii) if $X$ is a Banach space, $X$ has the R.D.P. and the cone $P$ is closed and generating, then $X$ has the C.P.P.

Proof. If $X$ has the C.P.P. then $Y$, ordered by the cone $P$, has the C.P.P. Let $Y$ have the C.P.P. If $x_{0} \in \operatorname{EP}(P)$, there exists a continuous positive projection $P_{x_{0}}(x)=\rho\left(x_{0}, x\right) x_{0}$ defined on $Y$. Let $\rho^{\prime}\left(x_{0},{ }^{\cdot}\right)$ be a Hahn-Banach extension of $\rho\left(x_{0}, \cdot\right)$ on $X$. Then $\rho^{\prime}\left(x_{0},{ }^{\circ}\right)$, is positive and $P_{x_{0}}^{\prime}(x)=\rho^{\prime}\left(x_{0}, x\right) x_{0}$ is a continuous projection of $x_{0}$ defined on $X$. Hence the statement (i) is true. To prove (ii) and (iii) we assume that the cone $P$ is closed and generating and that $X$ has the R.D.P. Since $P$ is closed $X$ is Archimedean. If $x_{0} \in \operatorname{EP}(P)$, by [8, Theorem 1.2], there exists a positive linear functional $f$ of $X$ such that

$$
f(x)=\sup \left\{t \in \mathbb{R}_{+} \mid t x_{0} \leqq x\right\}, \quad \forall x \in P .
$$

(In [8, Theorem 1.2], the existence of $f$ is deduced from the fact that $X$ is Archimedean and $X$ has the R.D.P.).

Let $P_{x_{0}}(x)=f(x) x_{0}, \forall x \in X$. Then $P_{x_{0}}$ is a linear positive projection and 
$P_{x_{0}}(x) \leqq x \forall x \in P$ because the cone $P$ is closed. To show that $P_{x_{0}}$ is continuous it is enough to show that $f$ is continuous. By $[3,3.5 .6$.], the statement (iii) is true.

If $X$ is a locally solid linear lattice then $X$ has the R.D.P. and the cone $P$ is generating and closed. Since $0 \leqq f(x) x_{0} \leqq x, \forall x \in P$, there exists $a \in \mathbb{R}_{+}$such that

$$
|f(x)| \leqq \frac{a}{\left\|x_{0}\right\|}\|x\|, \quad \forall x \in P .
$$

If $x \in X$ then

$$
|f(x)| \leqq\left|f\left(x^{+}\right)\right|+\left|f\left(x^{-}\right)\right| \leqq \frac{a}{\left\|x_{0}\right\|}\left(\left\|x^{+}\right\|+\left\|x^{-}\right\|\right) \leqq \frac{2 a^{2}}{\left\|x_{0}\right\|}\||x|\| \leqq \frac{2 a^{3}}{\left\|x_{0}\right\|}\|x\| .
$$

Hence the statement (ii) is true.

Lemma 3.1. Let $X$ be a normed space ordered by the cone $P, B$ a base for $P$ defined by $f \in X^{*}, Y=\mathbb{R} \times X$ be ordered by the cone $Y_{+}=\mathbb{R}_{+} \times P$ and $B^{\prime}$ be the base for $Y_{+}$ defined by the functional $f^{\prime}(\xi, x)=\xi+f(x)$. Then

(i) each extremal point $(\xi, 0)$ of $Y_{+}$has a continuous projection,

(ii) $x_{0} \in \operatorname{sep}(B)$ if, and only if, $\left(0, x_{0}\right) \in \operatorname{sep}\left(B^{\prime}\right)$,

(iii) for each $a \in \mathbb{R}_{+} \backslash\{0\}$ and $g \in X^{*}$ we have: $g$ is a negative-strongly exposing functional of $x_{0}$ in $B$ if, and only if, $g^{\prime}(\xi, x)=-a \xi+g(x)$ is a negative-strongly exposing functional of $\left(0, x_{0}\right)$ in $B^{\prime}$.

Proof. It is clear that the statement (i) is true.

Let $x_{0} \in \operatorname{sep}(B)$ and $g \in X^{*}$ be a negative-strongly exposing functional of $x_{0}$ in $B$. Then for each $a \in \mathbb{R}_{+} \backslash\{0\}$, the functional $g^{\prime}(\xi, x)=-a \xi+g(x)$, exposes $\left(0, x_{0}\right)$ in $B^{\prime}$ and $g^{\prime}\left(0, x_{0}\right)=0$. Let $\left(\xi_{v}, x_{v}\right) \in B^{\prime}$ be such that $g^{\prime}\left(\xi_{v}, x_{v}\right)=-a \xi_{v}+g\left(x_{v}\right) \rightarrow 0$. Since $g\left(x_{v}\right) \leqq 0$ we have that

$$
\xi_{v} \rightarrow 0 \text { and } g\left(x_{v}\right) \rightarrow 0 .
$$

Since $f^{\prime}\left(\xi_{v}, x_{v}\right)=\xi_{v}+f\left(x_{v}\right)=1$ we have that

$$
f\left(x_{v}\right) \rightarrow 1 \text {. }
$$

Then $g\left(x_{v} /\left(f\left(x_{v}\right)\right)\right) \rightarrow 0$, hence $x_{v} \rightarrow x_{0}$ and $\left(\xi_{v}, x_{v}\right) \rightarrow\left(0, x_{0}\right)$. So $\left(0, x_{0}\right) \in \operatorname{sep}\left(B^{\prime}\right)$ and $g^{\prime}$ is a negative-strongly exposing functional of $\left(0, x_{0}\right)$ in $B^{\prime}$.

Let $\left(0, x_{0}\right) \in \operatorname{sep}\left(B^{\prime}\right)$ and $h \in Y^{*}$ be a negative-strongly exposing functional of $\left(0, x_{0}\right)$ in $B^{\prime}$. Then there exist $a \in \mathbb{R}_{+} \backslash\{0\}$ and $g \in X^{*}$ such that $h(\xi, x)=-a \xi+g(x)$. It is clear that $g(x) \leqq 0 \forall x \in P$. If $x_{v} \in B$ and $g\left(x_{v}\right) \rightarrow g\left(x_{0}\right)=0$, then $\left(0, x_{v}\right) \in B^{\prime}$ and $h\left(0, x_{v}\right)=g\left(x_{v}\right) \rightarrow 0=$ $h\left(0, x_{0}\right)$, hence $x_{v} \rightarrow x_{0}$. So the statements (ii) and (iii) are true.

Theorem 3.1. Let $X$ be a normed space ordered by the cone $P, B$ be a base for $P$ 
defined by $f \in X^{*}, x_{0} \in \operatorname{sep}(B), g$ a negative-strongly exposing functional of $x_{0}$ in $B$ and $h=f-g$. Then:

(i) if $B^{\prime}$ is a base for $P$ defined by $f^{\prime} \in X^{*}$, and $y_{0}$ is an extreme point of $B^{\prime}$ with continuous projection $P_{y_{0}}$, then $y_{0} \in \operatorname{sep}\left(B^{\prime}\right)$ and $h_{y_{0}}$ strongly exposes $y_{0}$ in $B^{\prime}$.

(ii) the functional $h$ is uniformly monotonic and the cone $P$ is well-based.

Proof. Since $g$ is a negative-strongly exposing functional of $x_{0}$, we have that $-g$ is positive. Hence $h$ is strictly positive.

Proof of (i). By $\left(P_{3}\right), h_{y_{0}}$ exposes $y_{0}$ in $B^{\prime}$ and $h_{y_{0}}\left(y_{0}\right)=0$. Lét $\left(x_{v}\right)$ be a sequence of $B^{\prime}$ such that

$$
h_{y_{0}}\left(x_{v}\right)=h\left(P_{y_{0}}\left(x_{v}\right)-x_{v}\right) \rightarrow 0
$$

If $y_{v}=x_{v}-P_{y_{0}}\left(x_{v}\right)$, then $y_{v} \in P$ and

$$
-h_{y_{0}}\left(x_{v}\right)=h\left(y_{v}\right)=f\left(y_{v}\right)-g\left(y_{v}\right) \rightarrow 0
$$

Since $f\left(y_{v}\right),-g\left(y_{v}\right) \geqq 0$, we have that

$$
f\left(y_{v}\right) \rightarrow 0 \text { and } g\left(y_{v}\right) \rightarrow 0 .
$$

We put

$$
z_{v}=\left(1-f\left(y_{v}\right)\right) x_{0}+y_{v}
$$

Then there exists $v_{0} \in \mathbb{N}$ such that $z_{v} \in B$ for each $v \geqq v_{0}$. Since $g\left(z_{v}\right)=g\left(y_{v}\right) \rightarrow 0$ we have that $z_{v} \rightarrow x_{0}$, hence $y_{v} \rightarrow 0$. Since $f^{\prime}\left(x_{v}\right)=f^{\prime}\left(y_{v}\right)+f^{\prime}\left(P_{y_{0}}\left(x_{v}\right)\right)=1$, we have that $f^{\prime}\left(P_{y_{0}}\left(x_{v}\right)\right) \rightarrow 1$, hence, by $\left(P_{2}\right), P_{y_{0}}\left(x_{v}\right) \rightarrow y_{0}$. So $x_{v}=P_{y_{0}}\left(x_{v}\right)+y_{v} \rightarrow y_{0}$, hence $h_{y_{0}}$ strongly exposes $y_{0}$ in $B^{\prime}$.

Proof of (ii). Let $Y=\mathbb{R} \times X$ be ordered by the cone $Y_{+}=\mathbb{R}_{+} \times P$ and $B^{\prime \prime}$ be the base for $Y_{+}$defined by the functional $f^{\prime \prime}(\xi, x)=\xi+f(x)$. Then $\left(0, x_{0}\right) \in \operatorname{sep}\left(B^{\prime \prime}\right)$ and the functional $g^{\prime}(\xi, x)=-\xi+g(x)$ is a negative-strongly exposing functional of $\left(0, x_{0}\right)$ in $B^{\prime \prime}$.

If $h^{\prime}=f^{\prime \prime}-g^{\prime}$, then $h^{\prime}$ is strictly positive and

$$
h^{\prime}(\xi, x)=2 \xi+h(x)
$$

Let

$$
C=\left\{(\xi, x) \in Y_{+} \mid h^{\prime}(\xi, x)=1\right\}
$$

Then $z_{0}=(1 / 2,0)$ is an extreme point of $C$ with continuous projection $P_{z_{0}}(\xi, x)=(\xi, 0)$. 
By (i), $h_{z_{0}}^{\prime}$ strongly exposes $z_{0}$ in $C$. Moreover, for each $(\xi, x) \in C$ we have

$$
\left|h_{z_{0}}^{\prime}(\xi, x)\right| \leqq h^{\prime}(\xi, x)=1
$$

By Proposition 3.1, $C$ is bounded. So the base for $P$ defined by $h$ is bounded. Hence $P$ is well-based and the functional $h$ is uniformly monotonic.

Proposition 3.3. Let $X$ be a normed space ordered by the cone $P$ and $B$ be a base for $P$ defined by $f \in X^{*}$. If $h$ is a continuous uniformly monotonic linear functional of $X$, then for each extreme point $x_{0}$ of $B$ with continuous projection $P_{x_{0}}$, the functional $h_{x_{0}}$ strongly exposes $x_{0}$ in $B$.

Proof. Since $h$ is uniformly monotonic, there exists $a \in \mathbb{R}_{+} \backslash\{0\}$ such that $h(x) \geqq a\|x\|$, for each $x \in P$. Let $C=\{x \in P \mid h(x)=1\}$ and $x_{0}^{\prime}=\lambda x_{0} \in C$. To prove that $h_{x_{0}}$ strongly exposes $x_{0}$ in $B$, by Theorem 3.1, it is enough to prove that $h_{x_{0}}$ strongly exposes $x_{0}^{\prime}$ in $C$. Now $h_{x_{0}}$ exposes $x_{0}^{\prime}$ in $C$ and $h_{x_{0}}\left(x_{0}^{\prime}\right)=0$. Let $\left(x_{v}\right)$ be a sequence of $C$ such that

$$
h_{x_{0}}\left(x_{v}\right)=h\left(P_{x_{0}}\left(x_{v}\right)-x_{v}\right) \rightarrow 0 \text {. }
$$

Then $h\left(P_{x_{0}}\left(x_{v}\right)\right) \rightarrow 1$, hence

$$
P_{x_{0}}\left(x_{v}\right) \rightarrow x_{0}^{\prime}
$$

If $y_{v}=x_{v}-P_{x_{0}}\left(x_{v}\right)$, then $y_{v} \in P$ and

$$
h\left(x_{v}\right)=h\left(P_{x_{0}}\left(x_{v}\right)\right)+h\left(y_{v}\right)=1,
$$

hence $h\left(y_{v}\right) \rightarrow 0$. So we have that $y_{v} \rightarrow 0$, because $a\left\|y_{v}\right\| \leqq h\left(y_{v}\right)$. Hence $x_{v} \rightarrow x_{0}^{\prime}$.

Corollary 3.1. Let $X$ be a Banach space ordered by the closed, generating cone $P, B$ be $a$ base for $P$ and $X$ have the R.D.P. If $\operatorname{ep}(B) \neq \emptyset$ then the following statements are equivalent:

(i) $\operatorname{sep}(B) \neq \emptyset$,

(ii) $\operatorname{ep}(B)=\operatorname{sep}(B)$,

(iii) $P$ is well-based.

Proof. By Proposition $3.2 X$ has the C.P.P. Also by $[3,3.5 .6]$, each base for $P$ is defined by a continuous linear functional. By Theorem 3.1 we have that (i) $\Rightarrow$ (ii) and (i) $\Rightarrow$ (iii). It is clear that (ii) $\Rightarrow$ (i). If the cone $P$ is well-based, by $[3,3.8 .12]$, there exists a uniformly monotonic, continuous linear functional of $X$ and by Proposition 3.3 we have that (iii) $\Rightarrow$ (i).

Proposition 3.4. Let $X$ be a normed space ordered by the cone $P$ and $B$ be a base for $P$ defined by $f \in X^{*}$. If $x_{0}$ is an extreme point of $B$ with continuous projection $P_{x_{0}}$, then 
(i) $g$ is a negative-strongly exposing functional of $x_{0}$ in $B$ if, and only if, there exists a uniformly monotonic, continuous linear functional $h$ of $X$ such that $g=h\left(x_{0}\right) \rho\left(x_{0}, \cdot\right)-h$.

(ii) $g$ strongly exposes $x_{0}$ in $B$ if, and only if, there exists a uniformly monotonic, continuous, linear functional $h$ of $X$ and $\lambda \in \mathbb{R}$ such that $g=h\left(x_{0}\right) \rho\left(x_{0}, \cdot\right)-h+\lambda f$.

Proof. Let $h \in X^{*}$ be uniformly monotonic. By Proposition 3.3, $g=h_{x_{0}}=h\left(x_{0}\right) \rho\left(x_{0}, \cdot\right)-h$ is a negative-strongly exposing functional of $x_{0}$ in $B$. Let $g$ strongly exposes $x_{0}$ in $B$ and $g\left(x_{0}\right)=0$. By Theorem $3.1, h=f-g$ is uniformly monotonic. Let

$$
w=h-f+\rho\left(x_{0}, \cdot\right)
$$

Then $w\left(x_{0}\right)=1$ because $f\left(x_{0}\right)-h\left(x_{0}\right)=g\left(x_{0}\right)=0$. So

$$
g=w\left(x_{0}\right) \rho\left(x_{0}, \cdot\right)-w .
$$

To show that $w$ is uniformly monotonic it is enough to show that $w(x) \geqq \gamma>0$, $\forall x \in B^{\prime}=\{x \in P \mid h(x)=1\}$, because then $w(x) \geqq \gamma h(x) \geqq \gamma a\|x\| \forall x \in P$. Let $w\left(x_{v}\right) \rightarrow 0$ for a sequence $\left(x_{v}\right)$ of $B^{\prime}$. Then $w\left(x_{v}\right)=-g\left(x_{v}\right)+\rho\left(x_{0}, x_{v}\right) \rightarrow 0$ and therefore

$$
g\left(x_{v}\right) \rightarrow 0 \text { and } \rho\left(x_{0}, x_{v}\right) \rightarrow 0 .
$$

Moreover, $f\left(x_{v}\right) \rightarrow 1$ because $h\left(x_{v}\right)=f\left(x_{v}\right)-g\left(x_{v}\right)=1$. If $y_{v}=x_{v} /\left(f\left(x_{v}\right)\right)$ then $y_{v} \in B$ and $g\left(y_{v}\right) \rightarrow 0$. So $y_{v} \rightarrow x_{0}$, hence $x_{v} \rightarrow x_{0}$ and therefore $\rho\left(x_{0}, x_{v}\right) \rightarrow 1$. This is a contradiction, hence $w$ is uniformly monotonic and the proof of (i) is complete. The statement (ii) follows by (i) because $g$ strongly exposes $x_{0}$ in $B$ if, and only if, $g+\lambda f$ strongly exposes $x_{0}$ in $B$.

Proposition 3.5. Let $X$ be a normed space ordered by the cone $P$ and $B$ be a base for $P$ defined by $f \in X^{*}$. If $x_{0}$ is an extreme point of $B$ with continuous projection $P_{x_{0}}$ then the following statements are equivalent:

(i) $B$ is bounded,

(ii) for each sequence $\left(x_{v}\right)$ of $B, x_{v} \rightarrow x_{0}$ if, and only if, $P_{x_{0}}\left(x_{v}\right) \rightarrow x_{0}$.

Proof. Let $B$ be bounded. Then $f$ is uniformly monotonic and by Proposition 3.3, $f_{x_{0}}$ strongly exposes $x_{0}$ in $B$. Let $\left(x_{v}\right)$ be a sequence of $B$. If $x_{v} \rightarrow x_{0}$, then $P_{x_{0}}\left(x_{v}\right) \rightarrow P_{x_{0}}\left(x_{0}\right)=x_{0}$. If $P_{x_{0}}\left(x_{v}\right) \rightarrow x_{0}$, then

$$
f_{x_{0}}\left(x_{v}\right)=f\left(P_{x_{0}}\left(x_{v}\right)-x_{v}\right)=f\left(P_{x_{0}}\left(x_{v}\right)\right)-1 \rightarrow f\left(x_{0}\right)-1=0=f_{x_{0}}\left(x_{0}\right) .
$$

Hence $x_{v} \rightarrow x_{0}$. So (i) $\Rightarrow$ (ii).

Let the statement (ii) be true. To show that $B$ is bounded, it is enough to show that the functional $f_{x_{0}}$ which is bounded on $B$ strongly exposes $x_{0}$ in $B$. (Proposition 3.1.).

Let $\left(x_{v}\right)$ be a sequence of $B$ such that

$$
f_{x_{0}}\left(x_{v}\right) \rightarrow f_{x_{0}}\left(x_{0}\right)=0
$$


Then $f_{x_{0}}\left(x_{v}\right)=f\left(P_{x_{0}}\left(x_{v}\right)\right)-1 \rightarrow 0$, hence $P_{x_{0}}\left(x_{v}\right) \rightarrow x_{0}$ and $x_{v} \rightarrow x_{0}$. So $f_{x_{0}}$ strongly exposes $x_{0}$ in $\mathrm{B}$.

\section{Characterizations of $l_{1}(\Gamma)$}

Let $G$ be a closed and convex subset of a Banach space $X$. The set $G$ has the KreinMilman property (K.M.P.) if $K=\overline{\mathrm{co}} \mathrm{ep}(K)$, for each closed, convex and bounded subset $K$ of $G$. It is known, [2, 3.5.7], that the set $G$ has the Radon-Nikodým property (R.N.P.) if, and only if, $K=\overline{\operatorname{cosep}}(K)$, for each closed, convex and bounded subset $K$ of $G$. Moreover we know, [1], that in locally solid lattice Banach spaces the R.N.P. and the K.M.P. are equivalent. Let $\Gamma$ be any set. We denote by $l_{1}(\Gamma)$ the Banach space of all functions $\xi: \Gamma \rightarrow \mathbb{R}, \xi=(\xi(i))_{i \in \Gamma}$, such that $\sum_{i \in \Gamma}|\xi(i)|<+\infty$, with norm $\|\xi\|=\sum_{i \in \Gamma}|\xi(i)|$. The space $l_{1}(\Gamma)$ has the R.N.P., $[2,4.1 .9]$ and ordered by the cone $l_{1}^{+}(\Gamma)=$ $\left\{\xi \in l_{1}(\Gamma) \mid \xi(i) \geqq 0 \forall i \in \Gamma\right\}$ is a Banach lattice. The set $B=\left\{\xi \in l_{1}^{+}(\Gamma) \mid\|\xi\|=1\right\}$ is a closed bounded base for the cone $l_{1}^{+}(\Gamma)$. We denote by $l_{1}$ space $l_{1}(\mathbb{N})$.

Theorem 4.1. Let $X$ be an infinite-dimensional Banach space ordered by the closed, generating cone $P$ and $X$ have the R.D.P. Then:

(i) $X$ is order-isomorphic to $l_{1}(\Gamma)$ if, and only if, $P$ has a closed, bounded base with the K.M.P.;

(ii) $X$ is order-isomorphic to $l_{1}$ if, and only if, $P$ has a separable, closed, bounded base with the K.M.P.

Proof. Let $T$ be an order-isomorphism of $X$ onto $l_{1}(\Gamma)$. Since $B=\left\{\xi \in l_{1}^{+}(\Gamma) \mid\|\xi\|=1\right\}$ is a closed and bounded base for $l_{1}^{+}(\Gamma)$ with the K.M.P. we have that $T^{-1}(B)$ is a closed, bounded base for $P$ with the K.M.P. Let $B$ be a closed, bounded base for $P$ defined by the functional $f$ and let $B$ have the K.M.P. By $[3,3.5 .6]$, the functional $f$ is continuous. Let

$$
\operatorname{ep}(B)=\left\{b_{i} \mid i \in \Gamma\right\}
$$

By Proposition 3.2 we have that $X$ has the C.P.P.

We shall prove that

$$
x=\sum_{i \in \Gamma} \rho\left(b_{i}, x\right) b_{i} \quad \text { and } \quad \sum_{i \in \Gamma} \rho\left(b_{i}, x\right)<+\infty, \quad \forall x \in P
$$

At first we shall show that

$$
L=\left\{x \in P \mid P_{b_{i}}(x)=0, \quad \forall i \in \Gamma\right\}=\{0\} .
$$

The set $L$ is a cone and it is closed because $P_{b_{i}}$ is continuous $\forall i \in \Gamma$. If $L \neq\{0\}$, the set $B^{\prime}=$ $B \cap L$ is a non-empty, closed and bounded base for $L$. So $\operatorname{EP}(L) \neq \theta$ because $\operatorname{ep}\left(B^{\prime}\right) \neq 0$. Also $\operatorname{EP}(L) \subseteq \operatorname{EP}(P)$ because for each $x \in L$ and $y \in P, 0 \leqq y \leqq x$ implies that $y \in L$. Hence $b_{j} \in \operatorname{EP}(L)$ for at least one $j \in \Gamma$. This contradicts the definition of $L$ because $P_{b_{j}}\left(b_{j}\right)=b_{j}$. Hence $L=\{0\}$. 
We denote by $F$ the set of finite subsets of $\Gamma$ and for each $x \in P$ and $\delta \in F$ we denote by $x_{\delta}$ the sum

$$
x_{\delta}=\sum_{i \in \delta} P_{b_{i}}(x)
$$

Let $x \in B$. Then $\left(x_{\delta}\right)_{\delta \in F}$ is an upward-directed net of $P$ (if $\delta_{1}, \delta_{2} \in F$ we say that $\delta_{1} \leqq \delta_{2}$ if, and only if, $\left.\delta_{1} \subseteq \delta_{2}\right)$. We shall show that $x_{\delta}=\sup \left\{P_{b_{i}}(x) \mid i \in \delta\right\}$. If $z \geqq P_{b_{i}}(x) \forall i \in \delta$, then $w=z-P_{b_{i}}(x) \geqq 0$. If $j \in \delta$ and $j \neq i$, then $w \geqq P_{b_{j}}(w)=P_{b_{j}}(z) \geqq P_{b_{j}}(x)$ and therefore $z \geqq P_{b_{i}}(x)+$ $P_{b_{j}}(x)$. By a similar process we have that $z \geqq x_{\delta}$, hence

$$
x_{\delta}=\sup \left\{P_{b_{i}}(x) \mid i \in \delta\right\} \leqq x, \quad \forall \delta \in F
$$

By $[3,3.8 .8]$, we have

$$
y=\lim x_{\delta}=\sup _{\delta \in F}\left(x_{\delta}\right) \leqq x .
$$

This implies that $P_{b_{i}}(x) \leqq y \leqq x \forall i \in \Gamma$ and therefore that $P_{b_{i}}(x-y)=0 \forall i \in \Gamma$ because $P_{b_{i}}(x) \leqq P_{b_{i}}(y) \leqq P_{b_{i}}(x) \forall i \in \Gamma$. Hence $x=y$ and therefore

$$
x=\sum_{i \in \Gamma} \rho\left(b_{i}, x\right) b_{i}
$$

Since $f \in X^{*}$ and $f$ define the base $B$ we have that

$$
f(x)=\sum_{i \in \Gamma} \rho\left(b_{i}, x\right)=1
$$

So (1) is true because it is true for each $x \in B$.

We define the map $T: P \rightarrow l_{1}^{+}(\Gamma)$ as follows:

$$
T(x)=\left(\rho\left(b_{i}, x\right)\right)_{i \in \Gamma}, \quad \forall x \in P .
$$

It is clear that $T(\lambda x+\mu y)=\lambda T(x)+\mu T(y), \forall x, y \in P$ and $\lambda, \mu \in \mathbb{P}_{+} . T$ is one-to-one because

$$
x=\sum_{i \in \Gamma} \rho\left(b_{i}, x\right) b_{i}, \quad \forall x \in P
$$

Since the set $B$ is bounded there exists $M \in \mathbb{R}_{+}$such that

$$
\|x\| \leqq M, \forall x \in B
$$

We shall show that the map $T$ is onto.

Let $\xi=(\xi(i))_{i \in \Gamma} \in l_{1}^{+}(\Gamma)$. For each $\delta \in F$ we put

$$
x_{\delta}=\sum_{i \in \delta} \xi(i) b_{i} \quad \text { and } \quad \xi_{\delta}=T\left(x_{\delta}\right)
$$


Let $\varepsilon>0$. Since $\lim \xi_{\delta}=\xi$ there exists $\delta_{0} \in F$ such that

$$
\left\|\xi_{\delta_{1}}-\xi_{\delta_{2}}\right\|<\varepsilon, \quad \forall \delta_{1}, \delta_{2}>\delta_{0}
$$

If $\delta_{1}^{\prime}=\delta_{1} \backslash \delta_{2}$ and $\delta_{2}^{\prime}=\delta_{2} \backslash \delta_{1}$ then $\xi_{\delta_{1}^{\prime}}-\xi_{\delta_{2}^{\prime}}=\xi_{\delta_{1}}-\xi_{\delta_{2}}$. Since $\xi_{\delta_{1}^{\prime}}, \xi_{\delta_{2}^{\prime}}$ are disjoint we have that

$$
\left|\xi_{\delta_{1}}-\xi_{\delta_{2}}\right|=\left|\xi_{\delta_{1}^{\prime}}-\xi_{\delta_{2}^{\prime}}\right|=\xi_{\delta_{1}^{\prime}}+\xi_{\delta_{2}^{\prime}}
$$

and therefore

$$
\left\|\xi_{\delta_{1}}-\xi_{\delta_{2}}\right\|=\left\|\xi_{\delta_{1}^{\prime}}+\xi_{\delta_{2}^{\prime}}\right\|=\left\|\xi_{\delta_{1}^{\prime}}\right\|+\left\|\xi_{\delta_{2}^{\prime}}\right\| \leqq \varepsilon
$$

So

$$
\left\|x_{\delta_{1}}-x_{\delta_{2}}\right\|=\left\|x_{\delta_{1}^{\prime}}-x_{\delta_{2}^{\prime}}\right\| \leqq M\left(\left\|\xi_{\delta_{1}^{\prime}}\right\|+\left\|\xi_{\delta_{2}^{\prime}}\right\|\right)<M \varepsilon
$$

Hence the net $\left(x_{\delta}\right)_{\delta \in F}$ is Cauchy. If $x=\lim x_{\delta}$, then $x \in P$ and $\rho\left(b_{i}, x\right)=\xi(i) \forall i \in \Gamma$. So $T(x)=\xi$ and the map $T$ is onto $l_{1}^{+}(\Gamma)$. By $[3,1.5 .6] T$ can be extended to a linear map $T$ of $X$ into $l_{1}(\Gamma)$ as follows: $T(x)=T(y)-T(z)$, where $x=y-z$ and $y, z \in P$. Since $l_{1}^{+}(\Gamma)$ is generating the map $T$ is onto $l_{1}(\Gamma)$. Also $T$ is one-to-one because $T(x)=0$ implies $T(y)=T(z)$ and therefore $y=z$. By the definition of $T$ we have that $T$ and $T^{-1}$ are positive.

Let $\xi=T(x)=\left(\rho\left(b_{i}, x\right)\right)_{i \in \Gamma} \in l_{1}^{+}(\Gamma)$. Then

$$
\left\|T^{-1}(\xi)\right\|=\|x\|=\left\|\sum_{i \in \Gamma} \rho\left(b_{i}, x\right) b_{i}\right\| \leqq M \sum_{i \in \Gamma} \rho\left(b_{i}, x\right)=M\|\xi\| .
$$

Since the map $T^{-1}$ is linear and $l_{1}(\Gamma)$ is a Banach lattice we have that $T^{-1}$ is continuous. By the open mapping theorem, $T$ is continuous. So $T$ is an orderisomorphism of $X$ onto $l_{1}(\Gamma)$ and the statement (i) is true.

Let $B$ be a separable, closed and bounded base for $P$ and let $B$ have the K.M.P. Then $X$ is order-isomorphic to $l_{1}(\Gamma)$ and the base $C=T(B)$ for $l_{1}^{+}(\Gamma)$ is separable. Also there exists $\lambda \in \mathbb{R}_{+}$such that $\|\xi\| \geqq \lambda>0$ for each $\xi \in C$. Let $\operatorname{ep}(C)=\left\{\xi_{i} \mid i \in \Gamma\right\}$. Then

$$
\left\|\xi_{i}-\xi_{j}\right\|=\left\|\xi_{i}\right\|+\left\|\xi_{j}\right\| \geqq 2 \lambda, \forall i \neq j .
$$

So the set $\Gamma$ is countable because $C$ is separable and $\left\|\xi_{i}-\xi_{j}\right\| \geqq 2 \lambda, \forall i \neq j$. Hence $X$ is ordered isomorphic to $l_{1}$ and the statement (ii) is true.

Let $K$ be a closed, convex, unbounded subset of a Banach space $X$. For each real number $\rho>0$ we denote by $K_{\rho}, K_{s, \rho}$, the sets

$$
\{x \in K \mid\|x\| \leqq \rho\},\{x \in K \mid\|x\|=\rho\}
$$

respectively, whenever these sets are non-empty. In [7, Propositions 1 and 3] it is proved:

(i) if $X$ has the K.M.P. then $K_{\rho} \neq \overline{\operatorname{co}} K_{s, \rho}$ for at least one $\rho \in \mathbb{R}_{+}$implies $\operatorname{ep}(K) \neq \emptyset$; 
(ii) if $X$ has the R.N.P., then: $K_{\rho} \neq \overline{\operatorname{co}} K_{s, \rho}$ for at least one $\rho \in \mathbb{R}_{+} \Leftrightarrow K$ is dentable $\Leftrightarrow \operatorname{sep}(K) \neq \emptyset$.

It is easy to show that the proof of these results can be accepted for the case where $K$ is a subset of a closed, convex and unbounded subset $A$ of $X$ and $A$ has the K.M.P., the R.N.P. respectively.

In [7, Corollary 3], it is shown that each closed and convex subset of $l_{1}^{+}$has at least one strongly exposed point. In the following proposition we prove a similar result for well-based cones.

Proposition 4.1. Let $X$ be a Banach space ordered by the closed, well-based cone $P$ and $K$ a closed and convex subset of P. If $P$ has the K.M.P. (respectively, the R.N.P.) then $\operatorname{ep}(K) \neq \emptyset($ respectively, $\operatorname{sep}(K) \neq \emptyset)$.

Proof. If the set $K$ is bounded the proposition is true. Let $K$ be unbounded. To show that $\operatorname{ep}(K) \neq \emptyset$ (respectively, $\operatorname{sep}(K) \neq \emptyset$ ) it is enough to show that $\overline{c o} K_{s, \rho} \neq K_{\rho}$, for at least one $\rho \in \mathbb{R}_{+}$. Let a uniformly monotonic, continuous linear functional $f$ of $X(f(x) \geqq a\|x\|) \forall x \in P, x_{0} \in K$ and a real number $\varepsilon>0$. If $\rho>\left\|x_{0}\right\|$ and $a \rho>f\left(x_{0}\right)+\varepsilon$, then $x_{0} \in K_{\rho}$ and for each convex combination $x=\sum_{i=1}^{n} \lambda_{i} x_{i}$ of elements of $K_{s, \rho}$ we have

$$
f(x)=\sum_{i=1}^{n} \lambda_{i} f\left(x_{i}\right) \geqq \sum_{i=1}^{n} \lambda_{i} a\left\|x_{i}\right\|=a \rho>f\left(x_{0}\right)+\varepsilon .
$$

Hence for each $y \in \overline{\operatorname{co}} K_{s, \rho}$ we have that

$$
f(y) \geqq f\left(x_{0}\right)+\varepsilon>f\left(x_{0}\right)
$$

and therefore $\overline{\overline{c o}} K_{s, \rho} \neq K_{\rho}$.

Proposition 4.2. Let $X$ be an infinite-dimensional Banach space ordered by the closed, generating cone $P$ and $X$ have the R.D.P.

If $P$ has the K.M.P. the statements (i), (ii), (iii), (iv) and (v) are equivalent.

If $P$ has the R.N.P. all the following statements are equivalent:

(i) $X$ is order-isomorphic to $l_{1}(\Gamma)$,

(ii) $P$ is well-based,

(iii) $\operatorname{sep}(B) \neq \emptyset$, for at least one base $B$ for $P$,

(iv) $0 \in \operatorname{sep}(P)$,

(v) $\operatorname{sep}(K) \neq \emptyset$ for each closed and convex subset $K$ of $P$,

(vi) $B$ is dentable, for at least one base $B$ for $P$,

(vii) $P$ is dentable,

(viii) $K$ is dentable, for each closed and convex subset $K$ of $P$.

Proof. Let $P$ have the K.M.P. If $P$ is well-based, there exists a uniformly monotonic continuous linear functional $f$ of $X$. This functional defines a closed and bounded base 
$C$ for $P$ and $C$ has the K.M.P. Hence, by Theorem 4.1 , (ii) $\Leftrightarrow(i)$. Since $\operatorname{ep}(C) \neq \emptyset$, by Corollary 3.1 , we have that (ii) $\Leftrightarrow$ (iii). It is easy to show that (ii) $\Leftrightarrow$ (iv). Also (v) $\Rightarrow$ (iii) $\Rightarrow$ (ii). Since (ii) $\Leftrightarrow$ (i), the statement (ii) implies that $P$ has the R.N.P. and by Proposition 4.1 we have that (ii) $\Rightarrow$ (v).

Let $P$ have the R.N.P. Then for each closed and convex subset $A$ of $P$ we have: $\operatorname{sep}(A) \neq \emptyset$ if and only if, $A$ is dentable. Hence (iii) $\Leftrightarrow($ vi), (iv) $\Leftrightarrow$ (vii) and (v) $\Leftrightarrow$ (viii).

\section{REFERENCES}

1. J. Bourgain and M. Talagrand, Dans un espace de Banach reticulé solid, la propriété de Radon-Nikodým et celle de Krein-Milman sont équivalentes, Proc. Amer. Math. Soc. 81 (1981), 93-96.

2. R. D. Bourgin, Geometric Aspects of Convex Sets with the Radon-Nikodým Property (Lecture Notes in Mathematics 993).

3. G. J. O. Jameson, Ordered Linear Spaces (Lecture Notes in Mathematics, 141).

4. D. Kendall, Simplexes and vector lattices, J. London Math. Soc. 37 (1962), 365-371.

5. R. R. PhelPs, Dentability and extreme points in Banach spaces, J. Functional Analysis 16 (1974), 78-90.

6. I. A. Polyrakis, Lattice Banach spaces order-isomorphic to $l_{1}$, Math. Proc. Cambridge Phil. Soc. 94 (1983), 519-522.

7. I. A. Polyrakis, Extreme points of unbounded, closed and convex sets in Banach spaces, Math. Proc. Cambridge Phil. Soc. 95 (1984), 319-323.

8. G. C. SCHmidT, Extensions theorems for linear lattices with positive algebraic basis, Periodica Mathematica Hungarica 6(4), (1975), 295-307.

Department of Mathematics

National Technical University

ZOGRAFON CAMPUS, 15773

Athens, Greece 